\title{
In-situ Synchrotron Tensile Investigations on the Phase Responses within an Oxide Dispersion-strengthened (ODS) 304 Steel
}

\author{
Yinbin Miao ${ }^{\mathrm{a}, *}$, Kun $\mathrm{Mo}^{\mathrm{b}}$, Zhangjian Zhou ${ }^{\mathrm{c}}$, Xiang Liu ${ }^{\mathrm{a}}$, Kuan-Che Lan ${ }^{\mathrm{a}}$, Guangming Zhang ${ }^{\mathrm{a}, \mathrm{c}}$, \\ Michael K. Miller ${ }^{\mathrm{d}}$, Kathy A. Powers ${ }^{\mathrm{d}}$, Jonathan Almer ${ }^{\mathrm{e}}$, James F. Stubbins ${ }^{\mathrm{a}}$ \\ ${ }^{a}$ Department of Nuclear, Plasma, and Radiological Engineering, University of Illinois at Urbana-Champaign, Urbana, \\ IL 61801 \\ ${ }^{b}$ Nuclear Engineering Division, Argonne National Laboratory, Lemont, IL 60493 \\ ${ }^{c}$ Department of Material Science and Engineering, University of Science and Technology Beijing, Beijing, China, \\ 100084 \\ ${ }^{d}$ Center for Nanophase Materials Sciences, Oak Ridge National Laboratory, Oak Ridge, TN, 37830 \\ ${ }^{e} X$-ray Science Division, Argonne National Laboratory, Lemont, IL 60493
}

\begin{abstract}
Austenitic oxide dispersion-strengthened (ODS) alloys provide excellent mechanical strength and radiation tolerance along with their intrinsic advantages in corrosion resistance and high temperature creep resistance. This paper reports the in-situ synchrotron X-ray diffraction (XRD) tensile test results of ODS 304 stainless steel specimens. The oxygen-enriched nanoparticles were first characterized by both atom probe tomography (APT) and analytic scanning transmission electron microscopy (STEM). Three different types of precipitate phases were recognized, including large scale (around 100nm) TiN, intermediate scale (around 20nm) Y-Al-O, and small scale (< $5 \mathrm{~nm}$ ) Y-Ti-O. The lattice responses of different phases within the alloy to the externally applied stress indicates a prominent load partitioning phenomenon. This phenomenon was found to be highly dependent on the size of the precipitates. In addition, deformation-induced martensitic transformation was examined by the modified Williamson-Hall analyses of peak broadening, and was found to be different from that in ordinary 304 stainless steel.
\end{abstract}

Keywords: structure materials, oxide-dispersion strengthened steel, atom probe tomography, transmission electron microscopy, synchrotron, wide-angle X-ray scattering

\section{Introduction}

Sintering and heat treatment procedures following mechanical alloying of steel and oxide powders introduce oxygen-enriched nanoparticles into metallic matrices. These dispersive precipitates with extreme hardness can suppress dislocation gliding, significantly enhancing the yield and ultimate tensile strengths of ODS steels[1]. The nanoparticles also create many extra interfaces that can act as sinks for radiation-induced defects so that the radiation tolerance of ODS steels is improved[2]. Due to their characteristic superiority in mechanical strength, ferritic and martensitic

\footnotetext{
*Address: 216 Talbot Laboratory, 104 South Wright Street, Urbana, IL 61801 Tel: 217-607-6294 Email:
} miao2@illinois.edu 
steels have been the focus of existing ODS steel studies[3]. However, austenitic stainless steels, which are stable at elevated temperature, exhibit outstanding resistance to both corrosion and creep. The addition of oxygen-enriched nanoparticles can undoubtedly compensate the insufficiencies in mechanical strength and radiation resistance of austenitic steels, which makes austenitic ODS stainless steels promising for prospective applications as structural materials in advanced fission and fusion systems[4, 5, 6]. Recently, an ODS 304 steel was developed with the goal of improving the corrosion and creep resistance by introducing acceptable compromise in the mechanical strength and swelling resistance[7].

The properties of oxygen-enriched nanoparticles, including size distribution, chemical composition, and morphology, are the key factors that determine the performance of ODS alloys. Analytic scanning transmission electron microscopy (STEM), which is enhanced by the use of advanced spectroscopies such as electron dispersive X-ray spectroscopy (EDS) and electron energy loss spectroscopy (EELS), is an ideal way to examine the chemical composition of the medium-sized oxygen-enriched nanoparticles $(>5 \mathrm{~nm})[8,9]$. Atom probe tomography (APT) is capable of precisely capturing the elemental information of nanoclusters that are even smaller than $2 \mathrm{~nm}[10]$. Therefore, the combined application of analytic STEM and APT provides a unique and complementary method to obtain the chemical composition of oxygen-enriched nanoparticles with a wide range of dimensions. In addition, synchrotron X-ray diffraction (XRD), due to its high energy and high intensity, can yield information approximately the crystal structure of the nanoparticles[11].

In ODS steels, the strengthening mechanism depends significantly on the interactions between densely distributed nanoparticles and dislocations. Under deformation conditions, these interactions lead to the partitioning of load on the matrix and the precipitates. Being capable of monitoring the alterations in lattice parameters of multiple phases during tensile tests, in-situ XRD tensile testing is an ideal method to examine the load partitioning phenomenon. However, the low volume fraction of precipitate phases such as the oxygen-enriched nanoparticles in ODS steels makes it quite challenging for the conventional $\mathrm{X}$-ray source to form distinguishable diffraction peaks. Therefore, a synchrotron photon source, which features ultra high energies and ultra intensities, must be utilized. In fact, in-situ synchrotron X-ray tensile tests have been successfully employed to examine the strengthening contribution of precipitates in metallic materials, including carbides in F/M steels[7, 12, 13, 14, 15, 16] and Ni-based alloys[17], cementites in the ultrahigh-carbon steel[18], and oxides in the ODS ferritic steel[19]. Therefore, it is promising to use a similar technique to examine the behavior of dispersive precipitates in ODS steels.

The addition of Ni helps stabilize the austenite phase. However, the Ni content in stainless steel 304 is relatively low so that the austenite phase is only metastable at ambient temperature[20, 21]. That is, the austenite turns into martensite once the 304 steel is deformed, a process which is therefore called deformation-induced martensitic transformation. Previous studies on the 304 steel revealed two different types of transformation mechanisms[22]: one mechanism requires $\varepsilon$-martensite as the intermediate phase, whereas the other involves the direct transformation into $\alpha^{\prime}$-martensite. Both mechanisms are initiated by the formation of stacking faults and twinning faults. In FCC materials, stacking faults are the consequences of the dissociation of perfect dislocations, and twinning faults result from the accumulation of stacking faults[23]. Hence, both types of faults were dependent on the evolution of the dislocations. In the presence of densely distributed oxygen-enriched nanoparticles, the behavior of dislocations in ODS steels is quite different from those in conventional materials due to the complex interactions between the dislocations and nanoparticles[24, 25]. Therefore, it is worth exploring the deformation-induced martensitic transformation in ODS 304 steel. 


\section{Description of Experiments}

The ODS stainless steel 304 (Fe-18Cr-8Ni-1Mo-0.5Ti-0.15Si-0.35 $\left.\mathrm{Y}_{2} \mathrm{O}_{3}\right)$ was investigated in this study[7]. The base materials were mechanically alloyed in a nitrogen atmosphere using a planetary ball mill at $300 \mathrm{rpm}$ with a ball-to-powder mass ratio of 5:1 for 30 hours. The milled powders were then degassed, sealed, and consolidated by hot isostatic pressing (HIP) under a pressure of $100 \mathrm{MPa}$ at $1100^{\circ} \mathrm{C}$ for 2 hours and then at $1150^{\circ} \mathrm{C}$ for another hour. The as-HIPed sample was then forged at $1150^{\circ} \mathrm{C}$ with a forging ratio of $3: 1$. Additional hot rolling was performed three times with a reduction ratio of approximately $20 \%$ for each stage. These post-milling heat treatments ensure the great tensile elongation property of the specimen.

Specimens for atom probe tomography (APT) were fabricated from small blanks $(0.25 \mathrm{~mm}$ $\times 0.25 \mathrm{~mm} \times 10 \mathrm{~mm})$ that were cut from the bulk material. The blanks were electropolished into needle-shaped specimens with the use a standard loop method in a Simplex Electropointer and standard electrolytes[26]. The needle-shaped specimens were annular milled in a Dualbeam FEI Nova 200 Nanolab focused ion beam/scanning electron microscope (FIB/SEM) to ensure a circular cross section, as well as a suitable end radius and taper angle[27]. APT characterizations were performed in an energy-compensated CAMECA Instruments Inc. local electrode atom probe (LEAP ${ }^{\circledR} 4000 \mathrm{X}$ HR). Due to the poor electrical and thermal conduction of these materials, the specimens were analyzed in laser-mode at a specimen temperature of $30 \mathrm{~K}$, a pulse repetition rate of $200 \mathrm{kHz}$, a focused laser beam energy of either 50 or $100 \mathrm{pJ}$, and a data collection rate between $0.5 \%$ and $4 \%$ ions per field evaporation pulse depending on the standing voltage applied to the specimen. The position of the laser beam on the apex of the specimen was adjusted automatically during the experiment to account for the field evaporation of material from the apex of the specimen and specimen drift. These conditions resulted in individual LEAP ${ }^{\circledR}$ datasets containing up to 600 million atoms. Surface regions that contained damage from the gallium ion beam were not used for analysis. Data analysis was performed with the use of CAMECA Instruments Inc. Integrated Visualization and Analysis Software (IVAS 3.6.6). The proxigrams[28] were calculated according to the isosurfaces defined by $8 \%$ decomposed oxygen concentration. Thus, the full width at half maximum (FWHM) of the oxygen concentration in proxigrams was regarded as the size of the oxygen-enriched nanoclusters.

The in-situ synchrotron tensile tests were conducted at the 1-ID beamline of the Advanced Photon Source (APS) at Argonne National Laboratory (ANL). The material was cut into the ss-3 type tensile specimens with the gauge section of $1.49 \mathrm{~mm} \times 0.76 \mathrm{~mm} \times 7.60 \mathrm{~mm}$ by means of electrical discharge machining (EDM). Uniaxial tensile stress was applied to the specimen until failure. The experiment was conducted at ambient temperature. The diffraction analysis was performed with a monochromatic $70 \mathrm{keV}$ synchrotron beam with a $300 \mu \mathrm{m} \times 300 \mu \mathrm{m}$ cross-section. The "Hydra" detector array, which is composed of four identical GE angio type 2D detectors, was utilized to collect the diffraction signals. Nine points along the axial direction were scanned for each stress/strain state so as to enhance the accuracy of the measurement by improving statistics (see Fig. 1).

The $10^{\circ}$ region of the Debye-Scherrer rings near the tensile direction was integrated to give the lattice strain of the uniaxial direction, $\varepsilon_{11}=\left(d-d_{0}\right) / d_{0}$. The lattice strains of different phases were compared with macroscopic tensile behavior in order to reveal the load partitioning phenomenon. The volume fractions of matrices and precipitates were calculated by the following equation[22]: 


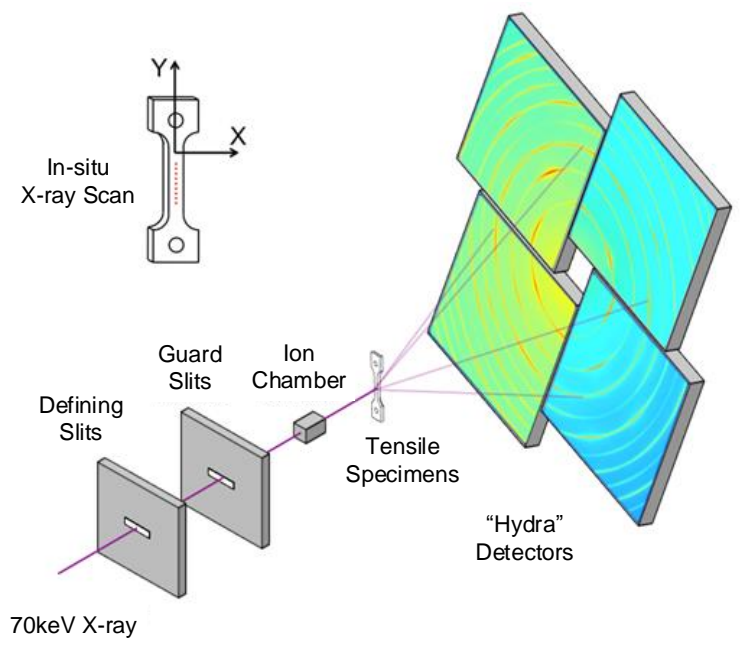

Figure 1: Experimental setup of the in-situ synchrotron X-ray diffraction tensile tests

$$
V_{i}=\frac{\frac{1}{n_{i}} \sum_{j=1}^{n_{i}} \frac{I_{i}^{j}}{R_{i}^{j}}}{\frac{1}{n_{a}} \sum_{j=1}^{n_{a}} \frac{I_{a}^{j}}{R_{a}^{j}}+\frac{1}{n_{m}} \sum_{j=1}^{n_{m}} \frac{I_{m}^{j}}{R_{m}^{j}}+\frac{1}{n_{p_{1}}} \sum_{j=1}^{n_{p_{1}}} \frac{I_{p_{1}}^{j}}{R_{p_{1}}^{j}}+\frac{1}{n_{p_{2}}} \sum_{j=1}^{n_{p_{2}}} \frac{I_{p_{2}}^{j}}{R_{p_{2}}^{j}}+\ldots}
$$

,where, $V_{i}$ is the volume fraction of phase $i$; $i$ represents austenite (subscript ${ }_{a}$ ), martensite (subscript ${ }_{m}$ ), and various precipitate phases (subscripts $p_{1}, p_{2}, \ldots$ ); $I_{i}^{j}$ is the intensity of the reflection of $j$ (for a specific $h k l$ ) for phase $i ; n_{i}$ is the number of the reflections for a certain phase; and $R_{i}^{j}$ is the material scattering factor for a specific phase $(i)$ and a reflection $(j)$, which has the expression as follows:

$$
R_{h k l}=\frac{1}{V^{2}}\left[|F|^{2} p\left(\frac{1+\cos ^{2} 2 \theta}{\sin ^{2} \theta \cos \theta}\right)\right] e^{-2 M}
$$

,where, $V$ is the volume of the unit cell; $F$ is the structure factor; $p$ is the multiplicity of the reflection; $\frac{1+\cos ^{2} 2 \theta}{\sin ^{2} \theta \cos \theta}$ is the Lorentz-polorization factor as a function of diffraction angle $\theta$; and $e^{-2 M}$ is the temperature factor, which was neglected in this room temperature study. Other information was retrieved by analyzing the breadth of XRD peaks according to the modified Williamson-Hall (W-H) method. The modified W-H analysis usually gives grain size and dislocation density of each phase. However, in the case of ODS 304, the evolution of stacking and twinning faults, which significantly contributes to the peak broadening, also plays an important role in plastic deformation and martensitic transformation. Therefore, a modified $\mathrm{W}-\mathrm{H}$ method that considers stacking and twinning faults was employed for the austenite phase:

$$
\Delta K=\left(\frac{1.5 \alpha+\beta}{a}\right) W(g)+\frac{0.9}{D}+\left(\frac{\pi A^{2} b^{2}}{2}\right)^{\frac{1}{2}} \rho^{\frac{1}{2}}\left(K \bar{C}^{\frac{1}{2}}\right)
$$


,where $\alpha$ is the stacking fault portion, $\beta$ is the twinning fault portion, $a$ is the lattice parameter, $W(g)$ is a reflection-dependent parameter given in Ref. [29], $D$ is the grain size, $A$ is an adjustable parameter that was chosen to be 2 for compatibility with a dislocation density of approximately $10^{15} \mathrm{~m}^{-2}, \bar{C}$ is a material parameter calculated according to Ungár et al.'s equations, $K=2 \sin \theta / \lambda$, $\Delta K=2 \cos \theta \Delta \theta / \lambda, \theta$ is the diffraction angle, and $\Delta \theta$ is the breadth of the peak. A pseudo-Voigt function was used for peak fitting. The pseudo-Voigt peak has two components:

$$
p V(2 \theta)=I_{0}[\eta L(2 \theta)+(1-\eta) G(2 \theta)]
$$

,where $I_{0}$ is the peak intensity, $L(2 \theta)$ is the Lorentzian component, $G(2 \theta)$ is the Gaussian component, and $\eta$ is the portion of the Lorentzian component. The breadth of the peak then has the following form:

$$
\Delta \theta=\omega\left[\pi \eta+(1-\eta)(\pi / \ln 2)^{1 / 2}\right]
$$

, where $\omega$ is the half of the peak's FWHM. The values of $\bar{C}$ for edge and screw dislocation are similar. Therefore, in this study, the dislocation density was calculated assuming all the dislocations are pure screw dislocations. This assumption does not influence the order of magnitude and the trend of the dislocation density.

The pre-strained ODS 304 specimens were mechanically thinned to $100 \mu \mathrm{m}$ and punched into $3 \mathrm{~mm}$ discs before being electropolished by $5 \%$ perchloric acid and $95 \%$ methanol at $-14^{\circ} \mathrm{C}$ using a Struer Tenupol-5 twin-jet polisher. The post-strained samples was lifted out from the gauge part of the tensile specimen by an FEI HELIOS 600i FIB. Diffraction contrast images were used to characterize the alterations in grain size, phase domination, and dislocation density after straining. STEM was utilized to examine the chemical compositions of the nanoparticles larger than $5 \mathrm{~nm}$ with the aid of electron dispersive X-ray spectroscopy (EDS). All the conventional TEM images were taken on a JEOL $2010 \mathrm{LaB}_{6}$ TEM, whereas the STEM-EDS data were collected on a JEOL 2010F EF-FEG TEM. In addition, a JEOL 7000F scanning electron microscope (SEM) was used to capture the images of the fracture surface of the post-tensile specimen.

\section{Results and Discussion}

\subsection{Phase identification}

The STEM high angle annual dark field (HAADF) technique provides Z-contrast images. As implied by its name, Z-contrast images are sensitive to the atomic numbers of materials. Therefore, it offers an efficient method for identifying different phases within one TEM sample. A typical STEM Z-contrast image of the pre-strained ODS 304 specimen is shown in Fig. 2. Three types of precipitates with various morphologies can be distinguished. One is the polygonal particles with large scale (around $100 \mathrm{~nm}$ ), the second is the nearly spherical particles with intermediate scale (around $20 \mathrm{~nm}$ ), and the third is particles with extremely small scale $(<5$ $\mathrm{nm})$. EDS analyses indicated that the polygonal particles are enriched in $\mathrm{Ti}$ and $\mathrm{N}$; while the intermediate phases are enriched in $\mathrm{Y}, \mathrm{Al}$ and $\mathrm{O}$. Aluminum was not added into the system intentionally. However, it is a common impurity in raw metal powders. The $\mathrm{Y} / \mathrm{Al}$ ratio measured by EDS showed the existence of both yttrium aluminum monoclinic (YAM), $\mathrm{Y}_{4} \mathrm{Al}_{2} \mathrm{O}_{9}$, and yttrium aluminum garnet (YAG), $\mathrm{Y}_{5} \mathrm{Al}_{3} \mathrm{O}_{12}$. The portion of YAM is much larger than that of YAG. STEM-EDS is incapable of analyzing the chemical composition of the extremely small precipitates due to the limitation of resolution and the contributions from the matrix. APT has 
atom-level resolution, and therefore was used to characterize these extremely small dispersive nanoclusters. APT succeeded in capturing these small nanoclusters and showed that they mainly contain Y, Ti and O. Those Y-Ti-O nanoclusters also contain low but non-negligible content of $\mathrm{Al}$ (see Fig. 3), which is consistent with Sakasegawa et al.'s findings in the ferritic ODS steel, MA957[30]. The volume fraction of these Y-Ti-O nanoclusters is $0.23 \%$ as determined by the APT data.

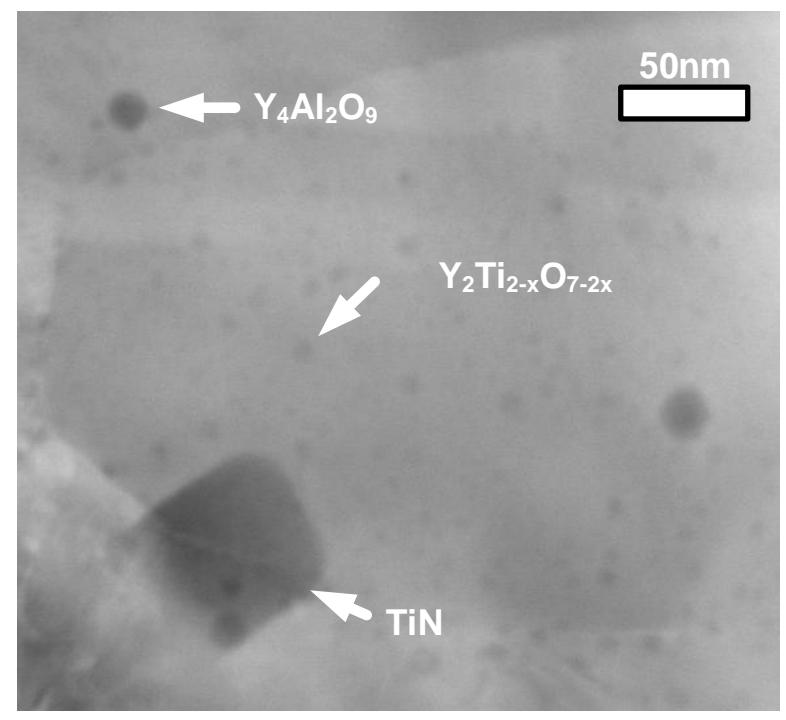

Figure 2: STEM HAADF images showing the existence of three precipitate phases: large scale TiN, intermediate scale Y-Al-O, and small scale Y-Ti-O

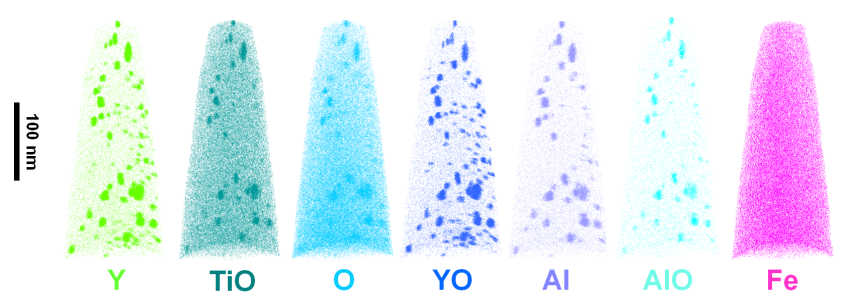

Figure 3: APT data showing the enrichment of $\mathrm{Y}, \mathrm{Ti}$, and $\mathrm{O}$ in small nanoclusters. Low but non-negligible content of $\mathrm{Al}$ was also found in these nanoclusters.

Synchrotron XRD results of the pre-strained sample are shown in Fig. 4. Based on the chemical composition information obtained by STEM-EDS and APT, two precipitate phases can be identified: TiN and YAM. TiN has a typical $\mathrm{NaCl}$ structure, and its strongest reflection was found to be (111); in the contrast, the strongest reflections of YAM are $(023) /(032)$ reflection and (221) reflection, both of which show similar intensity (Fig. 4). According to previous studies, 
Y-Ti-O nanoclusters smaller than $2 \mathrm{~nm}$ lack a crystal structure, but large Y-Ti-O particles have a stoichiometry of $\mathrm{Y}_{2} \mathrm{Ti}_{2-x} \mathrm{O}_{7-2 x}$, which has a pyrochlore structure[31, 32]. Therefore, Y-Ti-O nanoparticles contribute either no peaks or peaks of the pyrochlore structure. Due to the low volume fraction of Y-Ti-O, it is common that only the strongest peak, (222), is distinguishable. Unfortunately, the d-spacing of (222) reflection is approximately $2.95 \AA$, close to the $\mathrm{d}$-spacings of the dual reflections of YAM. Since the intensity of YAM reflections is higher, the (22 $\overline{1})$ reflection of Y-Ti-O cannot be identified. Therefore, in this synchrotron study, only two precipitate phases, TiN and YAM, were analyzed and discussed. The volume fractions of TiN and YAM are $0.65 \pm 0.03 \%$ and $0.66 \pm 0.03 \%$, respectively, according to the calculation based on Equation 1. In addition, minor peaks from the martensitic phase were also identified in the pre-strained sample. This was the consequence of the deformation-induced martensitic transformation during the manufacturing process.

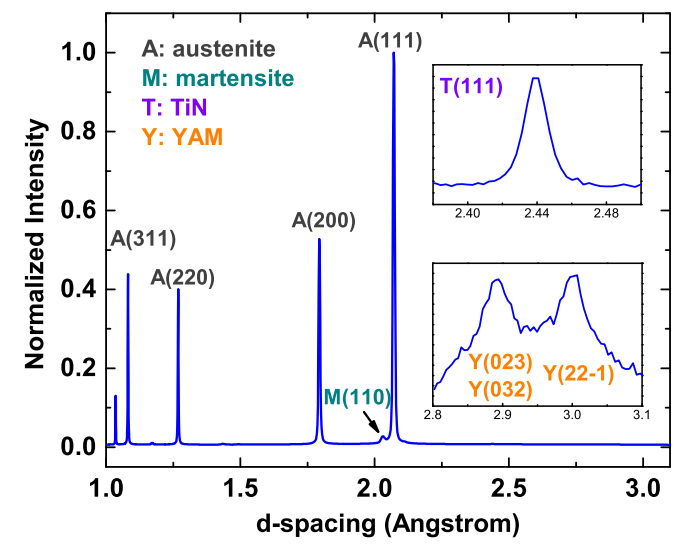

Figure 4: Synchrotron X-ray diffraction line profile of the pre-strained ODS 304 specimen: two matrix phases, austenite and martensite, along with two precipitate phases, TiN and YAM, can be recognized

\subsection{Load partitioning phenomenon}

The strain-stress diagram of the ODS 304 specimen was measured during in-situ synchrotron tensile tests, and is shown in Fig. 5. Each point represents a set of synchrotron measurements. The yield strength (YS) and the ultimate tensile strength (UTS) are $852 \mathrm{MPa}$ and $946 \mathrm{MPa}$, respectively. Both values are far higher than those of non-ODS austenitic steels[20, 21]. The elongation of ODS 304 steel is around 42\%, showing that this steels maintains excellent ductility but with a significant improvement in mechanical strength.

The d-spacing evolutions of all distinguishable phases were analyzed. Due to the anisotropic moduli, the lattice strains vary among the different diffraction conditions. Some specific diffractions yield lattice strains similar to the macroscopic strain. The d-spacing evolution of all distinguishable phases were analyzed. For a specific phase, different reflections can behave diversely with applied stresses, not only due to the anisotropic elastic moduli, but also as a result of the microstructural development (e.g. dislocation structure evolution) during the deformations. Previous studies 


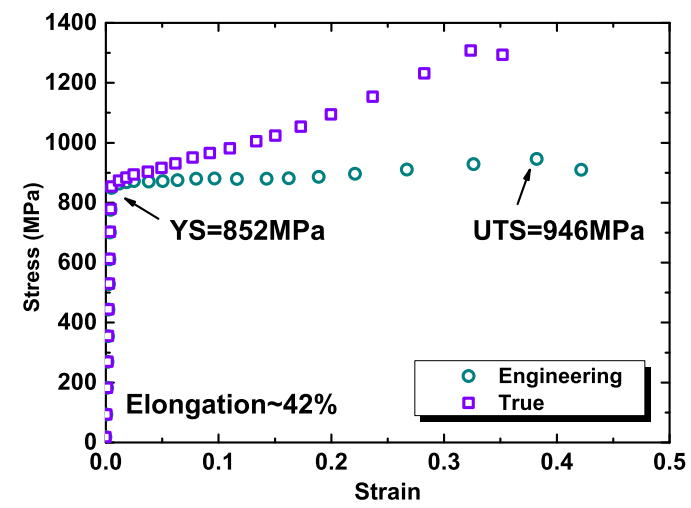

Figure 5: Strain-stress diagram of the in-situ synchrotron tensile test: the yield strength and ultimate tensile strength of ODS 304 are significantly enhanced compared to ordinary 304 steel, with little compromise in ductility

confirm that the (311) reflection is a suitable representation for characterization of macroscopic stresses and strains for face-centered cubic (FCC) metals[33, 17]. From a recent study in a ferritic/martensitic ODS steel, the loading behavior of different reflections show little difference during the entire tensile test[19]. Among all reflections of the martensitic matrix, the elastic constant of (321) reflection was found to approach the value of the bulk material[34]. Therefore, the (311) reflection of the austenitic matrix and the (321) reflection of the martensitic matrix were selected to represent the matrix phases. For the precipitate phases, there exist a limited number of distinguishable diffraction peaks. Thus, (111) of TiN and (22) $)$ of YAM were chosen to represent the lattice strain of precipitates. The behavior of the lattice strain alteration in response to an increasing macroscopic stress is illustrated in Fig. 6. It is obvious that all the phases have very similar lattice strains in the elastic deformation regime. However, the lattice strain of YAM experiences a slight rise as the specimen approaches yielding. Once yielded, a prominent load partitioning phenomenon can be observed: the lattice strains of YAM and TiN become much higher than those of the matrices. Comparing macroscopic strain with lattice strains (see Fig. 7) provides a different insight into the load partitioning phenomenon: within the elastic regime, lattice strains of all the phases are comparable to the macroscopic strain. As the specimen yields, lattice strains become lower than the macroscopic strain since dislocation gliding, namely, plasticity, begins to account for a significant share of deformation. The Young's moduli of the four distinguishable phases with specific orientations can be derived from the stiffness tensors of these phases: $E_{a}(311)=201 \mathrm{GPa}[35], E_{m}(321)=279 \mathrm{GPa}[36], E_{t}(111)=337$ $\mathrm{GPa}[37]$, and $E_{y}(22 \overline{1})=199 \mathrm{GPa}[38]$ ( $t$ for TiN, and $y$ for YAM). Based on these moduli, the elastic stresses of all the four distinguishable phases were calculated. The elastic stress vs. true strain diagram, as shown in Fig. 8, indicates the load partitioning phenomenon more clearly by directly providing the load situation of each phase.

Since there are two different synchrotron recognizable precipitate phases existing in the ODS 304 steel, it is interesting to compare the behaviors of TiN and YAM. In the STEM images, TiN particles were observed to be much larger and sparser than YAM ones. Also, the YAM 


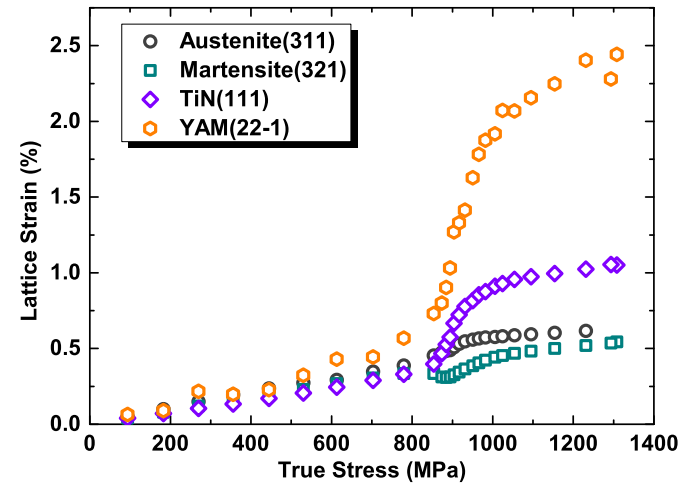

Figure 6: Lattice strain vs. true stress for all the distinguishable phases within ODS 304 steel

phase take more stress after yielding compared with TiN. Here, a very strong size effect on the load partitioning phenomenon is revealed. This size effect is due to the different interaction mechanisms of particles of different sizes with dislocations[39]. This size effect also implies that those Y-Ti-O nanoclusters, which have smaller size and are indistinguishable in synchrotron $\mathrm{XRD}$, are supposed to take even more stress than the YAM particles. In fact, the ultra-fine $\mathrm{Y}_{2} \mathrm{Ti}_{2} \mathrm{O}_{7}$ has been reported to take higher load compared to TiN in a strained $9 \mathrm{Cr}$ ferrite ODS steel[19], in which YAM precipitates are absent.

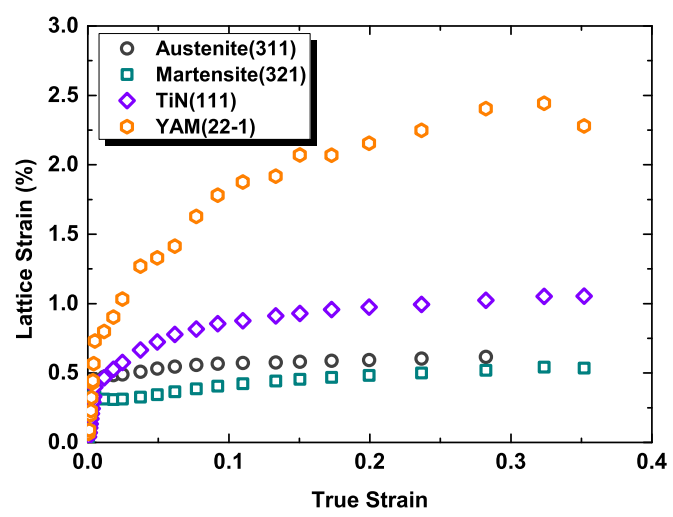

Figure 7: Lattice strain vs. true strain for all the distinguishable phases within ODS 304 steel

The SEM image of the fracture surface taken from the post-tensile specimen, Fig. 9, illustrates the formation of dense distributions of submicron-scale voids that cause the failure. It is worth mentioning that large and medium oxygen-enriched nanoparticles can be identified in some of 


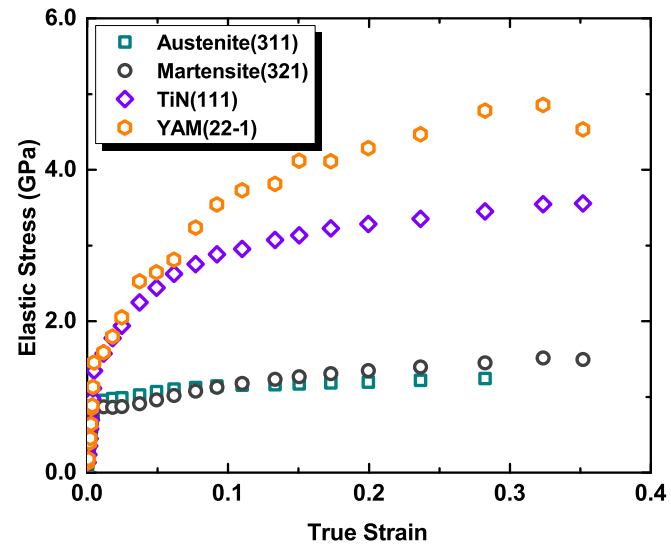

Figure 8: Elastic stress vs. true strain for all the distinguishable phases within ODS 304 steel

these voids.

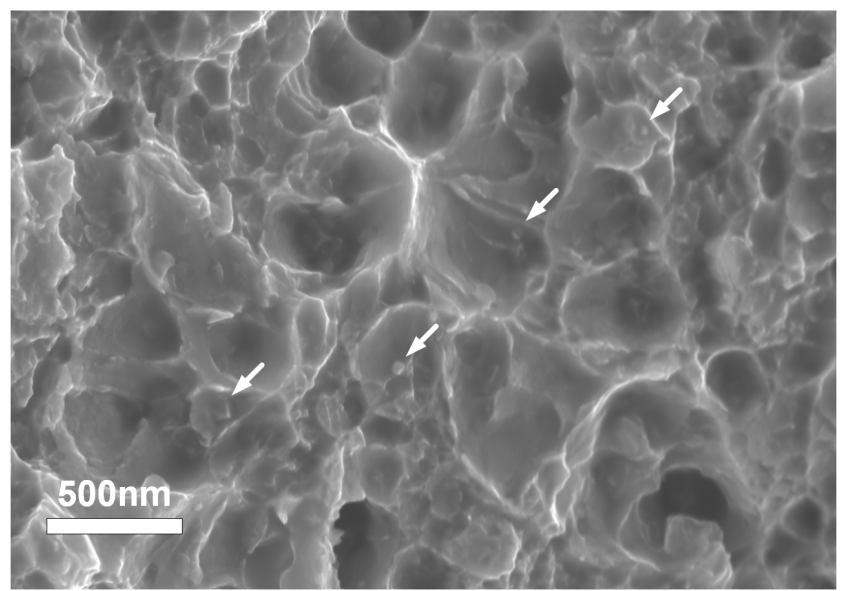

Figure 9: SEM image of the fracture surface: some large and medium oxygen-enriched nanoparticles (marked by arrows) are distinguishable on the fracture surface

\subsection{Deformation-induced martensitic transformation}

Even in the presence of oxygen-enriched nanoparticles, the deformation-induced martensitic transformation of the austenitic matrix is still prominent. By comparing the TEM bright field images of both pre-tensile and post-tensile specimens, shown in Figs. 10 and 11, it is obvious that the dominant phase of the pre-tensile specimen is austenite, the majority of which transforms 
into martensite at failure. These TEM images also indicate that the grain sizes of both initial austenite grains and final martensite grains are around hundreds of nanometers.

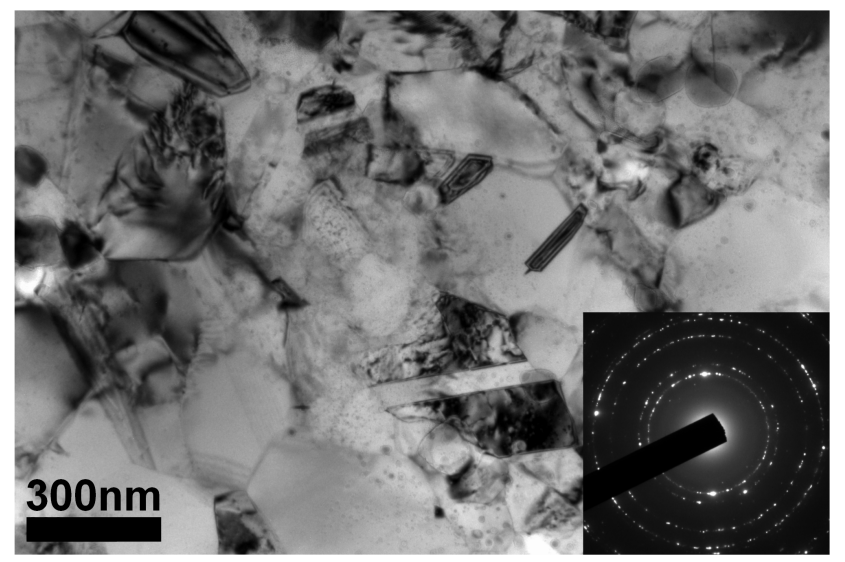

Figure 10: TEM bright field image of the pre-tensile specimen. The average grain size is around several hundred nanometers. Dense dispersive oxide nanoparticles are observable, and the dislocation density is low.

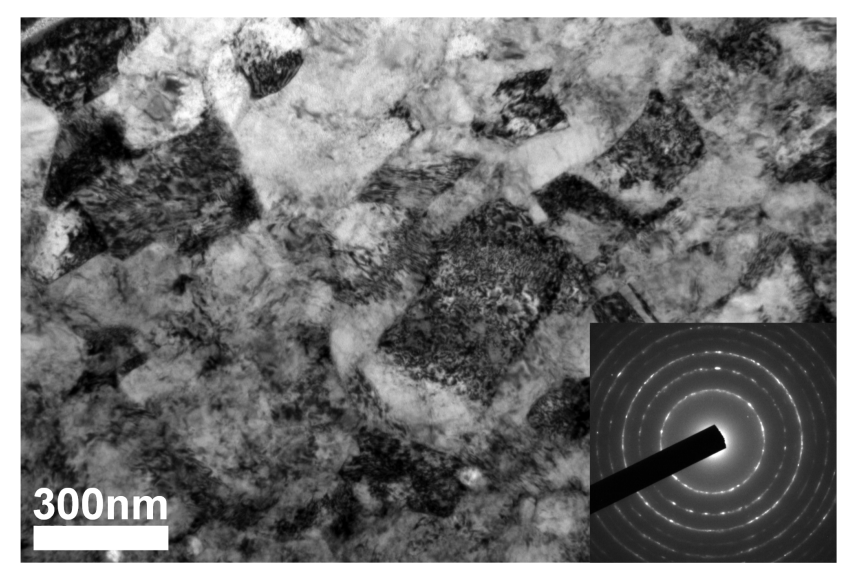

Figure 11: TEM bright field image of the post-tensile specimen. The average grain size is around several hundred nanometers, and the dislocation density is high

The deformation-induced martensitic transformation was monitored by calculating the volume fractions of both austenitic and martensitic phases at each measurement point. The strain-induced martensitic transformation begins during the elastic regime. However, the phase transition is quite marginal before yielding. Once the plastic deformation starts, the transformation becomes significant. In fact, as the specimen begins necking, over $90 \%$ of the austenitic matrix has turned into martensite (see Fig. 12). All the martensite observed in this study is $\alpha^{\prime}$-martensite. No $\epsilon$-martensite was ever distinguished in the synchrotron XRD data, implying that the direct $\gamma-\alpha^{\prime}$ phase transformation mechanism is dominant throughout the tensile test. 


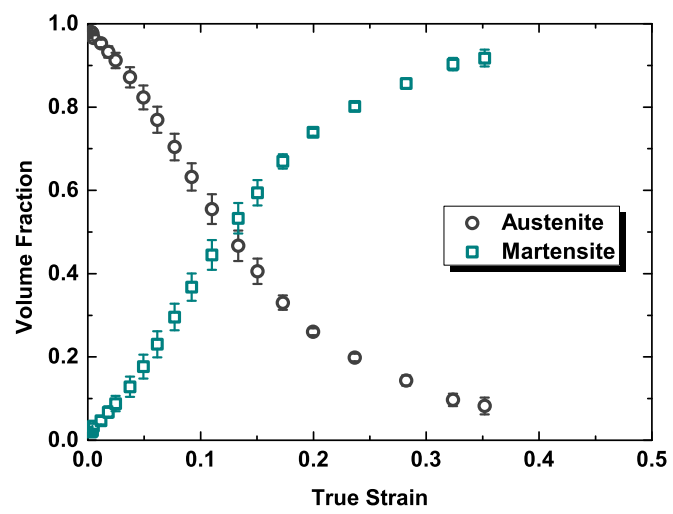

Figure 12: Volume fraction evolution of austenitic and martensitic matrices. The initial martensite percentage is around $2 \%$ and the final martensite percentage is over $90 \%$

\subsection{Modified Williamson-Hall analyses}

The changes in grain sizes of both austenite and martensite matrices are shown in Fig. 13 . The grain size measured by a modified W-H method is only meaningful when it is smaller than several hundred nanometers. In addition, this measurement is only accurate within an order of magnitude and for a correct trend of evolution. The initial austenite grain size is around hundreds of nanometers, which is consistent with the TEM observation, shown in Fig. 10. This value begins to decrease even during the elastic regime. This phenomenon is consistent with the fact that the martensitic transformation begins during elastic deformation (as shown in Fig. 12). At the end of the tensile tests, the average grain size of austenite is approximately $10 \mathrm{~nm}$, and the grains exist as small residual austenite grains. On the other hand, the initial grain size of martensite is around $20 \mathrm{~nm}$. The martensite grains continue to grow throughout the tensile test. At failure, the martensite grains are as large as several hundreds of nanometers, also consistent with the TEM observation, shown in Fig. 11.

The modified W-H method also provided information on the portion of stacking faults and twinning faults in austenite matrix (Fig. 14). There existed some stacking and/or twinning faults in the original specimens, but these faults vanish during elastic deformation. As the deformation-induced martensitic transformation is initiated by stacking and twinning faults, this phenomenon may explain the martensitic transformation during the elastic regime. The stacking and twinning faults then decrease to an indistinguishable level in the early stages of plastic deformation. Meanwhile, the martensitic transformation continues, indicating that the newly formed stacking and twinning faults can rapidly turn into $\alpha^{\prime}$-martensite. In the late stages of plastic deformation, the portion of stacking and twinning faults finally rises and then decreases again as the austenite phase is depleted, which is consistent with Shen et al.'s findings in non-ODS 304 steel[23].

Lastly, the evolution of dislocation densities is illustrated in Fig. 15. In austenite, the dislocation density has a peak value of $1.4 \times 10^{15} \mathrm{~m}^{-2}$, and then decreases to a marginal value as the austenite phase becomes a minor matrix phase. On the other hand, the dislocation density in 


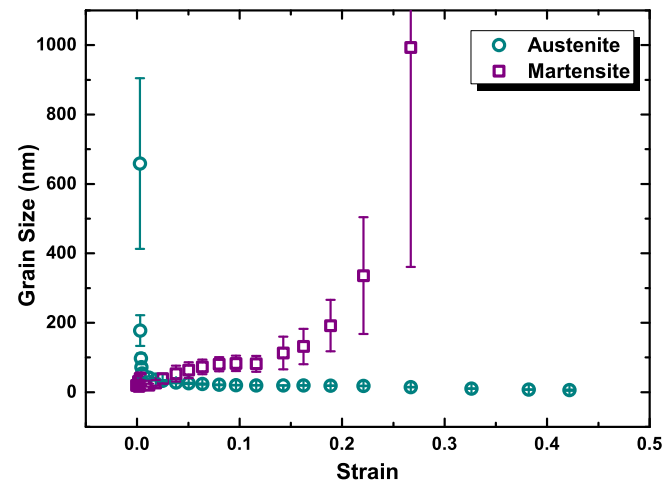

Figure 13: Grain size evolution in matrix phases

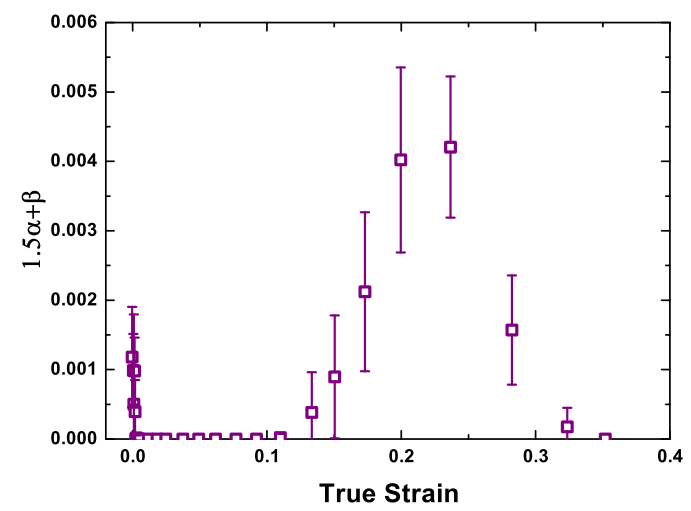

Figure 14: Stacking fault and twinning fault evolution in matrix phases 
martensite continues to increase throughout the entire plastic deformation regime. The maximum dislocation density, $3.0 \times 10^{15} \mathrm{~m}^{-2}$, is comparable to the measurement of the TEM image, $1.6 \times 10^{15}$ $\mathrm{m}^{-2}$, validating the selection of the fitting parameters. The dislocation density in martensite displays a trough-like behavior in the early stages of plastic deformation. This trough also corresponds to the fastest martensitic transformation rate shown in Fig. 12. A fast martensitic transformation creates a great number of martensitic grains with few dislocations, which significantly decreases the average dislocation density significantly. It is noticeable that the prominent increase of stacking and twinning faults only happens while the dislocation density in austenite is beyond a value of $7.6 \times 10^{14} \mathrm{~m}^{-2}$, showing an almost proportional relation between the dislocation behaviors and stacking/twinning fault formation.

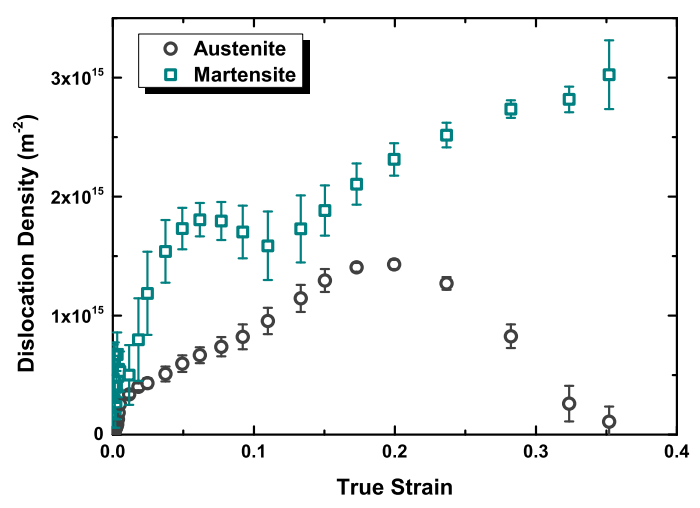

Figure 15: Dislocation density evolution in matrix phases

Previous room temperature and sub- $0{ }^{\circ} \mathrm{C}$ temperature tensile tests of ordinary 304 steels show the existence of both direct $\gamma-\alpha^{\prime}$ and indirect $\gamma-\varepsilon-\alpha^{\prime}$ mechanisms[22, 23]. However, in this room temperature tensile test of the ODS 304 steel, even the ultra-sensitive synchrotron XRD technique did not detect the formation of any $\varepsilon$-martensite. The absence of one martensitic transformation mechanism implies the effect of the oxygen-enriched nanoparticles. The $\varepsilon$-martensite nucleation always takes place in the regions that contain irregularly spaced stacking faults, whereas the direct nucleation of $\alpha^{\prime}$-martensite is usually related to the pile-ups of dislocations [40, 41, 42]. Therefore, in the ordinary 304 steel, the dislocation density is low in the early stages of plastic deformation. Due to the low stacking fault energy (SFE) of 304 steel at room temperature, the sparse dislocations can easily evolve into spaced stacking faults, which is the precursor of $\varepsilon$-martensite nucleation. On the contrary, the accumulation of dislocations leads to the formation of dense dislocation pile-ups in the late stages of plastic deformation, resulting in the dominance of direction nucleation of $\alpha^{\prime}$-martensite. This explanation is consistent with Shen et al.'s experimental observations[23]. However, in the presence of dense and dispersive distributions of nano-scale precipitates with excellent mechanical strength, as in ODS steels, the dislocation gliding is significantly suppressed. As a result, dislocation pile-ups are common even in the early stages of plastic deformation. Therefore, the $\gamma-\alpha^{\prime}$ mechanism is dominant immediately following yielding. 


\section{Conclusion}

Multiple advanced characterization techniques were employed to study various phases within an ODS 304 stainless steel. The existence of two matrix phases, austenite and martensite, along with three precipitate phases, TiN, Y-Al-O and Y-Ti-O particles, was confirmed by a combination of STEM-EDS and APT analyses. Except Y-Ti-O, both matrices and two precipitate phases were distinguishable in synchrotron XRD, and their responses to the tensile deformation were further investigated. Based on the observation on the microstructural development during the tensile test, the following conclusions can be drawn from this study:

1. YAM takes much more loads than TiN, showing a strong size effect of the load partitioning phenomenon.

2. The deformation-induced martensitic transformation eliminates austenitic phase with dense dislocations and produces martensite with few dislocations, significantly influencing the grain size and dislocation density evolution during the tensile test.

3. The deformation-induced martensitic transformation in ODS 304 steel was found to be quite different from that in ordinary 304 steel, which is a consequence of fast dislocation pile-up buildup due to the existence of densely and dispersedly distributed oxygen-enriched nanoparticles as obstacles of dislocation gliding.

\section{Acknowledgement}

This work was supported by 973 DOE INL 120293. The TEM experiments were carried out in part in the Frederick Seitz Materials Research Laboratory Central Facilities, University of Illinois, which is partially supported by the U.S. Department of Energy under grants DEFG02-07ER46453 and DE-FG02-07ER46471. Atom probe tomography (APT) was conducted at the Center for Nanophase Materials Sciences, which is a DOE Office of Science User Facility. MKM was sponsored by the Materials Sciences and Engineering Division, Office of Basic Energy Sciences, US Department of Energy. Argonne National Laboratory's work was supported by U.S. DOE under Contract No. DE-AC02-06CH11357.

\section{References}

[1] M. S. El-Genk, J.-M. Tournier, Journal of Nuclear materials 340 (2005) 93-112.

[2] G. Odette, M. Alinger, B. Wirth, Annu. Rev. Mater. Res. 38 (2008) 471-503.

[3] S. Ukai, M. Fujiwara, Journal of Nuclear Materials 307 - 311, Part 1 (2002) 749 - 757.

[4] H. Oka, Y. Yamazaki, H. Kinoshita, N. Hashimoto, S. Ohnuki, S. Yamashita, S. Ohtsuka, in: MRS Proceedings, volume 1298, Cambridge Univ Press, pp. mrsf10-1298.

[5] H. Oka, M. Watanabe, H. Kinoshita, T. Shibayama, N. Hashimoto, S. Ohnuki, S. Yamashita, S. Ohtsuka, Journal of Nuclear Materials 417 (2011) 279-282.

[6] H. Oka, M. Watanabe, N. Hashimoto, S. Ohnuki, S. Yamashita, S. Ohtsuka, Journal of Nuclear Materials (2013).

[7] M. Wang, Z. Zhou, H. Sun, H. Hu, S. Li, Materials Science and Engineering: A 559 (2013) 287-292.

[8] A. Certain, K. G. Field, T. R. Allen, M. Miller, J. Bentley, J. Busby, Journal of Nuclear Materials 407 (2010) $2-9$.

[9] M. C. Brandes, L. Kovarik, M. K. Miller, M. J. Mills, Journal of Materials Science 47 (2012) 3913-3923.

[10] M. K. Miller, C. M. Parish, Materials Science and Technology 27 (2011) 729-734.

[11] Y. Miao, K. Mo, B. Cui, W.-Y. Chen, M. Miller, K. Powers, M. V., G. D., J. Almer, I. Robertson, J. F. Stubbins, Mater. Char. (under review).

[12] X. Pan, X. Wu, X. Chen, K. Mo, J. Almer, D. R. Haeffner, J. F. Stubbins, Journal of Nuclear Materials 398 (2010) $220-226$.

[13] X. Pan, X. Wu, K. Mo, X. Chen, J. Almer, J. Ilavsky, D. R. Haeffner, J. F. Stubbins, Journal of Nuclear Materials 407 (2010) 10-15. 
[14] L. Wang, M. Li, J. Almer, Acta Materialia 62 (2014) 239-249.

[15] M. Li, L. Wang, J. D. Almer, Acta Materialia 76 (2014) $381-393$.

[16] P. Mosbrucker, D. Brown, O. Anderoglu, L. Balogh, S. Maloy, T. Sisneros, J. Almer, E. Tulk, W. Morgenroth, A. Dippel, Journal of Nuclear Materials 443 (2013) 522-530.

[17] K. Mo, H.-M. Tung, J. Almer, M. Li, X. Chen, W. Chen, J. B. Hansen, J. F. Stubbins, Journal of Pressure Vessel Technology 135 (2013) 021502.

[18] M. Young, J. Almer, M. Daymond, D. Haeffner, D. Dunand, Acta materialia 55 (2007) 1999-2011.

[19] K. Mo, Z. Zhou, Y. Miao, D. Yun, H.-M. Tung, G. Zhang, W. Chen, J. Almer, J. F. Stubbins, Journal of Nuclear Materials 455 (2014) 376-381.

[20] P. L. Mangonon, G. Thomas, Metallurgical transactions 1 (1970) 1587-1594.

[21] P. L. Mangonon, G. Thomas, Metallurgical transactions 1 (1970) 1577-1586.

[22] A. K. De, D. C. Murdock, M. C. Mataya, J. G. Speer, D. K. Matlock, Scripta Materialia 50 (2004) 1445-1449.

[23] Y. Shen, X. Li, X. Sun, Y. Wang, L. Zuo, Materials Science and Engineering: A 552 (2012) 514-522.

[24] D. Häussler, B. Reppich, M. Bartsch, U. Messerschmidt, Materials Science and Engineering: A 309 (2001) 500-504.

[25] S. Ukai, S. Ohtsuka, T. Kaito, H. Sakasegawa, N. Chikata, S. Hayashi, S. Ohnuki, Materials Science and Engineering: A 510 (2009) 115-120.

[26] M. K. Miller, Atom probe tomography: Analysis at the atomic level, Springer, New York, NY, 2000.

[27] M. K. Miller, K. F. Russell, K. Thompson, R. Alvis, D. J. Larson, Microscopy and Microanalysis 13 (2007) $428-436$.

[28] O. C. Hellman, J. A. Vandenbroucke, J. Rüsing, D. Isheim, D. N. Seidman, Microscopy and Microanalysis 6 (2000) $437-444$.

[29] T. Ungár, in: Materials Science Forum, volume 278, Trans Tech Publ, pp. 151-157.

[30] H. Sakasegawa, F. Legendre, L. Boulanger, M. Brocq, L. Chaffron, T. Cozzika, J. Malaplate, J. Henry, Y. De Carlan, Journal of Nuclear Materials 417 (2011) 229-232.

[31] M. Tamura, H. Sakasegawa, K. Shiba, H. Tanigawa, K. Shinozuka, H. Esaka, Metallurgical and Materials Transactions A 42 (2011) 2176-2188.

[32] K. R. Whittle, M. G. Blackford, R. D. Aughterson, G. R. Lumpkin, N. J. Zaluzec, Acta Materialia 59 (2011) 7530-7537.

[33] B. Clausen, T. Lorentzen, T. Leffers, Acta Materialia 46 (1998) 3087-3098.

[34] K. Mo, A. Oaks, Y. Miao, J. F. Stubbins, http://materials.npre.illinois.edu/calculator/website.html (2013).

[35] L. Vitos, P. A. Korzhavyi, B. Johansson, Nature materials 2 (2002) 25-28.

[36] H. Zhang, B. Johansson, L. Vitos, Physical Review B 79 (2009) 224201.

[37] M. Zhang, J. He, Surface and Coatings Technology 142 (2001) 125-131.

[38] X. Zhan, Z. Li, B. Liu, J. Wang, Y. Zhou, Z. Hu, Journal of the American Ceramic Society 95 (2012) 1429-1434.

[39] D. Chrzan, J. Morris, Y. N. Osetsky, R. Stoller, S. Zinkle, MRS bulletin 34 (2009) 173-177.

[40] T. Suzuki, H. Kojima, K. Suzuki, T. Hashimoto, M. Ichihara, Acta Metallurgica 25 (1977) 1151-1162.

[41] J. Brooks, M. Loretto, R. Smallman, Acta Metallurgica 27 (1979) 1829-1838.

[42] J. Brooks, M. Loretto, R. Smallman, Acta Metallurgica 27 (1979) 1839-1847. 